\section{Bewährte Partnerschaft}

- Novartis Pharma und LETI Pharma haben die Verlängerung ihrer bereits seit 2005 bestehenden vertraglich vereinbarten Zusammenarbeit bis 2022 beschlossen. Grund hierfür sind die positiven Ergebnisse der bisherigen Kooperation zwischen dem breit aufgestellten internationalen Konzern und dem auf den Allergiebereich spezialisierten spanischen Mittelständler. Gleichzeitig haben die beiden Firmen ein umfangreiches Programm an Zulassungsstudien aufgesetzt. Damit kommen die Unternehmen den Anforderungen der Therapieallergene-Verordnung von 2008, die eine Ausdehnung der Vorschriften zur Arzneimittelzulassung auf Therapieallergene vorsieht, in vollem Umfang nach.

Novartis Pharma ist Meister in der klinischen Forschung: 2009 belegte das Unternehmen mit 115 Studien zuzüglich 37 Phase-II- und IIlb-Studien mit deutlichem Abstand Platz 1 bei der Anzahl der klinischen Studien in Deutschland. Über 16.000 Patienten waren in die Studien eingeschlossen, davon rund 2.000 aus dem Indikationsbereich Dermatologie/Pneumologie. Auf dem Gelände des Novartis-Partners LETI Pharma nahe Madrid wird ständig an Innovationen gearbeitet. Depigoid $^{\oplus}$ und Depiquick ${ }^{\circledR}$ werden mit einem einzigartigen Aufreinigungsverfahren - der sogenannten Depigmentierung - hergestellt. Damit werden allergologisch irrelevante Verunreinigungen besonders sorgfältig entfernt, was bei guter Verträglichkeit die Verabreichung hoher Allergendosen in kurzer Zeit ermöglicht. Darüber hinaus betreibt das Unternehmen in seiner Einrichtung in Spanien auch das weltweit größte Milbenzuchtlabor. red

Nach Informationen von Novartis Pharma und LETI Pharma
Neues Präparat bei leichter Neurodermitis

Für leichte Formen der Neurodermitis gibt es ab sofort eine neue, kortisonfreie Behandlungsoption in der Apotheke: Bepanthen Sensiderm Creme. Mit der hautverwandten Derma-MembranStruktur stabilisiert sie die Hautschutzbarriere, gleicht das Lipiddefizit in der Hornschicht aus und lindert so gerötete, juckende Hautstellen. Das Präparat enthält außerdem das bewährte Dexpanthenol.

Bepanthen Sensiderm Creme ist frei von Kortison, Emulgatoren sowie Duft-, Farbund Konservierungsstoffen. Daher ist sie besonders verträglich und eignet sich sowohl für die empfindliche Gesichtshaut als auch für Babys und Kinder, Schwangere und stillende Mütter - auch in der Langzeitanwendung. Das Präparat kann auf unverletzter und behaarter Haut angewendet werden und ist seit Januar rezeptfrei in der Apotheke erhältlich.

Nach Informationen von Bayer Vital

\section{Innovative Behandlung von Herpesbläschen}

Das neue Herpatch Pflaster ist eine echte Innovation bei der Behandlung von Herpesbläschen: Das Pflaster ist extrem dünn und legt sich wie ein unsichtbarer Schutzfilm über die infizierten Hautareale. Es bindet Wasser und bildet so um die entzündete Hautstelle eine physikalische Barriere - das Wundmilieu wird so positiv beeinflusst, Symptome wie Juckreiz, Schwellungen und Rötungen können schneller abklingen. Es beschleunigt auBerdem die Heilung der Bläschen, lindert den Schmerz und löst sich nach wenigen Stunden vollständig auf. Dadurch muss das Pflaster nicht abgezogen werden und das schmerzhafte Aufreißen der Herpesbläschen wird vermieden.

Wichtig: Damit das Pflaster gut haftet, muss das Patch im nassen Zustand angewandt werden. Am besten wird es dazu vor dem Auftragen für wenige Sekunden in Wasser getaucht und dann nass auf die Herpesbläschen aufgebracht. Die Hautstelle sollte gereinigt bzw. mit Wasser abgespült werden. Herpatch ist seit Mitte Dezember exklusiv in Apotheken zum Preis von 12,95 Euro erhältlich.

Nach Informationen von Deutsche Chefaro Pharma GmbH
Arzneimittel zum Auftragen auf die KopfAnwendung von 5 \%iger Minoxidil-Lösung (Regaine ${ }^{\circledast}$ Männer) im Unterschied zu den kosmetischen Präparaten evidenzbasiert pharmazie empfohlen. Auch bei DermatoEmpfehlungsrate.

ZurTherapie der androgenetischen Alopezie liche Beweis der Wirksamkeit erbracht worwohl bei Männern als auch bei Frauen (Regaine Frauen mit 2\% Minoxidil) die topische lust stoppen kann und in vielen Fällen sogar die Haardichte verbessert. Für andere lokal wirksame Arzneimittel dagegen sind die Wirksamkeitsbelege nur wenig überzeugend und Kosmetika sowie Nahrungsergänzungsmittel sind bei androgenetischer Alopezie wirkungslos - zu diesem Schluss kommt auch der aktuelle Öko-Test Ratgeber Gesundheit \& Fitness 2010.

red 